\title{
EIN ERSTER VERSUCH EINER TYPOLOGIE DER DEUTSCHEN ZEICHNUNG DES 19. JAHRHUNDERTS*
}

\author{
von WERNER BUSCH
}

Handzeichnungsforschung scheint in der Krise, sie ist schier inexistent. Es gibt sie nicht als eine das Medium, die Funktion und besonders die je historisch eigene Form der Weltbewältigung reflektierende, strukturell analysierende Methode. Sicher gibt es immer noch Kenner, wenn sie offenbar leider auch immer weniger werden, sicher auch gibt es vorzügliche Katalog- und Corpusarbeit, aber Handzeichnungswissenschaft gibt es nicht. Ausnahmen bestätigen die Regel, nur an eine sei erinnert. Alexander Perrigs Versuch einer Morphologie der Handzeichnung in seinen MichelangeloStudien von $1976^{1}$ hat keine Nachfolge gefunden. Aufbauend auf Degenhardts Bemerkungen zur Graphologie $^{2}$ entwarf Perrig eine Gestaltlehre, die sich Rechenschaft gibt über den körpermotorischen Vorgang bei der Formwerdung der Handzeichnung. Daraus wollte er Kriterien für Authentizität entwickeln. Offenbar war der Anspruch zu hoch, und das Projekt mußte stocken aus demselben Grund, aus dem das Rembrandt Research Project in die Krise geraten ist. In beiden Fällen sind die Detailergebnisse bewundernswert, der Ermessensspielraum konnte eingeengt, jedoch eben nicht, wie man wohl gehofft hatte, ausgeschaltet werden. Einer Typologie auf der Basis struktureller Analyse geht es nicht um Authentizität, sondern allein um historisches Verständnis. Was ist eine Zeichnung auf der jeweiligen historischen Stufe, was kann sie, deren funktionaler Zusammenhang jeweils neu zu klären ist, auf welche Weise veranschaulichen? Wie verarbeitet sie Wirklichkeit, wie nimmt sie Stellung zu Erfahrenem? Eine Typologie, die jeweils für regionale und historische Räume zu erstellen wäre, könnte uns helfen, Möglichkeiten der Wirklichkeitsaneignung des Mediums Handzeichnung zu erkennen, zu scheiden und in ihren methodischen Konsequenzen zu cha-

\footnotetext{
* Der vorliegende Beitrag erschien zuerst in der Festschrift Konrad Hoffmann, Zeitenspiegelung, Berlin 1998, S. 181-192.

1 Alexander Perrig, Michelangelo Studien I, Michelangelo und die Zeichnungswissenschaft. Ein methodologischer Versuch, Frankfurt/Bern 1976.

2 Bernhard Degenhart, Zur Graphologie der Handzeichnung. Die
}

rakterisieren. Drei Beispiele seien vorgeführt, wobei dem Autor die Vorläufigkeit des Vorgeschlagenen bewußt ist.

\section{ASMUS JAKOB CARSTENS}

Auf seiner Zeichnung »Priamos bittet Achill um den Leichnam Hektors" (Abb.1) von 1794 verschränkt Carstens auf besondere Weise Flächeund Raumerfahrung. Eigentliches Thema jedoch ist die Dissoziierung der Figuren im Raum. Carstens Komposition ist trotz der stark fluchtenden Raumkonstruktion völlig statisch. Dieser Effekt wird durch den auf die Symmetrieachse hin konstruierten Kastenraum erreicht, der nahsichtig genommen, mit dem Lineal konstruiert ist. Symmetrie legt Bewegung still. Zudem ist dieser Kastenraum insofern ein abstraktes Gebilde, als die Figuren nicht wirklich nach den Regeln der Zentralperspektive in ihm ihren Ort gefunden haben. ${ }^{5}$ Er ist ihr Gehäuse, behaust sie aber nicht. Der Fluchtpunkt des rigiden Konstruktes liegt leicht oberhalb des Kopfes von Priamos. Der Kopf des Achill dagegen ist so weit oberhalb der Horizontlinie angeordnet, wie der Kopf des Priamos sich unter ihr befindet. Dadurch werden ihre Isolierung vom übrigen Geschehen und zugleich der Unterwerfungsakt des Priamos anschaulich, der allerdings auch dadurch augenfällig wird, daß seine Figur sich vollständig unterhalb der durch die rechte Fußbodenleiste markierten Fluchtlinie befindet. Das Oval seines Gewandes bindet ihn zusätzlich in eine in sich geschlossene Grundform. Da Achill, wie die hinter ihm Stehenden, nicht über die vom linken oberen Wandband angegebene Fluchtlinie ragt, ist diese Linie so etwas wie eine ungestörte Bahn für den sinnenden Blick des melancholisch gebeugten Achill. Die

Strichbildung als stetige Erscheinung innerhalb der italienischen Kunstkreise, in: Kunstgeschichtliches Jahrbuch der Biblioteca Hertziana 1, 1937, S. 223-340.

3 Zum Kastenraum als neoklassizistischem Typus: Robert Rosenblum, Transformations in Late Eighteenth Century Art, ${ }^{3}$ Princeton 1970 , S. $189-191$. 


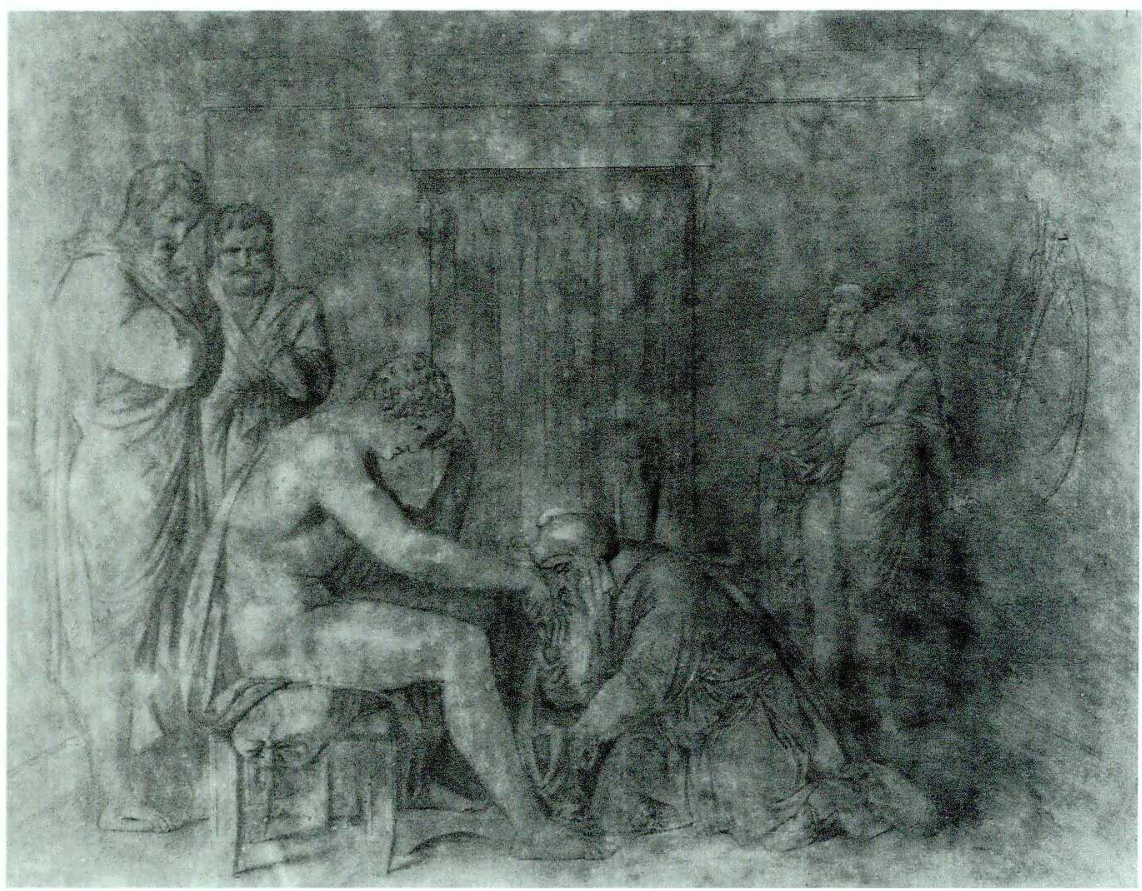

Abb. 1 Asmus Jakob Carstens, Priamos bittet Achill um den Leichnam Hektors, RöteI weiß3 gehöht über Graphit, 1794, 49,5 x 65,2 cm, Staatliche Museen zu Berlin, Kupferstichkabinett

Konstruktion dient aber auch dem übrigen Personal als abstraktes Zuordnungssystem. So sind die nahe hinter Achill stehenden Griechen dennoch zugleich weit von ihm entfernt, weil sie der hinteren Raumecke zugeordnet sind; der erste blickt parallel zur Rückwand, der andere mit dem Rücken zur Rückwand frontal zum Betrachter, sie wiederholen also den Raumwinkel. Durch ihre en face- und Profilanordnung sind sie wiederum voneinander isoliert, selbst in sich versunkene Reflexionsfiguren. Das jüngere Griechenpaar rechts erscheint, gemessen an der Raumkonstruktion, als zu weit entfernt. Sinnstiftend wird diese Unstimmigkeit insofern, als so die Trauer des Achill nicht gestört, von der Unterwerfung nicht abgelenkt wird. Das Paar ist der Rückwand zugeordnet, der Seitenkontur der Frau fällt mit der rechten Zimmerecke zusammen. Beide sind frontal dem Betrachter zugewandt, nur ihr Schatten an der rechten Seitenwand markiert den Winkel. Sie reflektieren das Geschehen nicht, wie die Alten hinter Achill, sondern merken auf, halten allerdings in der Bewegung inne; gemeint ist damit offenbar eine ihrem Alter gemäße Reaktion. Insgesamt gesehen haben wir einen Moment vollständiger Ruhe vor uns, bei dem man, auch im Sinne der Geschichte, nicht weiß, wie es weitergeht. Eigentliches Thema ist das Nachsinnen über ein un-

4. Zum Zusammenhang von Handlungslosigkeit, Stilisierung und Reflexion: Werner Busch, Das sentimentalische Bild. Die Krise der Kunst im 18. Jahrhundert und die Geburt der Moderne, München 1993 , bes. S. $137-180$. ausweichliches Schicksal, in dem die Polaritäten nicht aufgehoben sind, sondern anschaulich bleiben. Fläche ist gegen Raum gestellt, Fülle gegen Leere, Orthogonale gegen Diagonale. Barocke organische Bewegung, räumliche Entfaltung, Handlungsentwicklung, zielgerichtete Impulse, Aktion und Reaktion gibt es nicht mehr wirklich. Dafür eröffnet die Stillstellung für den Betrachter Projektions- und Reflexionsräume, er hat die Brüche im Kontinuum selbst zu ertragen, mit seiner Projektionsleistung zu überbrücken. ${ }^{4}$

Carstens' Freund und römischer Mitbewohner Fernow scheint indirekt die Gründe für diesen Effekt am präzisesten angegeben zu haben. 1806 in seiner Biographie von Carstens gibt er zu bedenken: »... dass unser Zeitalter ... eben so wenig mehr durch christliche als durch heidnische Mitologie zu begeistern ist; dass also auch beide, in Hinsicht auf religiöses Interesse, der Kunst gleich ferne liegen; so wie der seit gestern Todte, so todt ist wie der, welcher vor Jahrhunderten starb ...«. ${ }^{5}$ Ferner weist er darauf hin: »... dass Vergangenheit nie wieder Gegenwart werden kann, und dass es eben so unmöglich sein würde, die Kunst wieder zu ihrer einfältigen Kindheit, als unsere Zeit zu dem kindischen Geist und Glauben der Zeiten zurückzuführen, der jene entwickelt hat ... ${ }^{6}{ }^{6}$ Damit ist dreierlei konsta-

5 Carl Ludwig Fernow, Leben des Künstlers Asmus Jakob Carstens. Ein Beitrag zur Kunstgeschichte des achtzehnten Jahrhunderts, Leipzig 1806, S. 250.

6 Ebd., S. 250 f. 
tiert: 1. Die Gegenwart steht nicht mehr in religiöser Tradition. 2. Der Bruch mit der Vergangenheit ist unüberbrückbar und deshalb 3. sind zwar die alten Griechen, wie Carstens es tut, darzustellen, aber nicht mehr von exemplarisch verbindlicher Bedeutung. Thr Stil kann adaptiert werden, ihr Sinn ist neu zu verfügen, wir können ergänzen, individuell in der Projektionsleistung des Betrachters. Das heißt: 1. Die historische Dimension der Form wird offenbar, deswegen erscheint sie verfremdet, und 2. die Verzeichnungen, Raumbrüche, Ableseambivalenzen, paradoxen Verschränkungen von Raumund Flächeerfahrung dienen der mehr oder weniger abstrakt ausgelösten Wirkung. Der Betrachter wird die Wirkung als psychologische Befrachtung an den Gegenstand zurückgeben. Die Einsicht in den Vergangenheitscharakter von Kunst und Geschichte ist also gekoppelt an die Einsicht in die Subjektivität und Relativität des rückwärts gewandten Blickes, in die Unvermeidlichkeit der individuellen Aktivierung des Vergangenen. Chladenius hat schon Mitte des 18. Jahrhunderts geschrieben, der Historiker gebe die Geschichte in »verjüngten Bildern« wieder, an denen der Anteil der Gegenwart unübersehbar sei. ${ }^{7}$ Kommunikationsstörung scheint das Hauptthema der Kunst von Carstens zu sein; da im Bilde nicht überbrückt, sucht der Betrachter sie für sich durch Sinnbeimessung erträglich zu machen. Er also hat das Werk zu vollenden.

\section{CASPAR DAVID FRIEDRICH}

Die Zeichnung tritt bei Friedrich im Gegensatz zu Carstens entschieden nicht mit Autonomieanspruch auf, sie hat allein Studienmaterial zu liefern. Da sie unmittelbar dem Naturbild folgt, muß sie, ins Bild übernommen, transformiert werden. Das mag man idealistisch im klassischen Sinne nennen: das Studienmaterial wird, indem es einer Vorstellung, einer Idee folgt, entmaterialisiert. Doch im Gegensatz zur klassischen Tradition gibt es bei Friedrich im Normalfall nicht den fertigen Entwurf, den, in Schwinds Sinn, notfalls auch ein anderer ins Bild setzen könnte. Im Gegenteil, die farbige Fassung ist eben nicht eine Materialisierung der im Entwurf schon vollständigen Idee, sondern erst der Prozeß des farbigen Malens, als ein Prozeß der Selbsterfahrung, bewahrt den Naturvorwurf in einer Neustiftung auf, hebt ihn dialektisch auf.

Überschaut man Friedrichs Handzeichnungsœuvre, so stellt man doch mit einem gewissen Er-

7 J. M. Chladenius, Einleitung zur richtigen Auslegung vernünftiger Reden und Schrifften, Leipzig $1742, \$ 355$; s. dazu etwa Reinhart Koselleck, Vergangene Zukunft. Zur Semantik geschichtlicher Zeiten, Frankfurt a. M. 1989, S. 52.

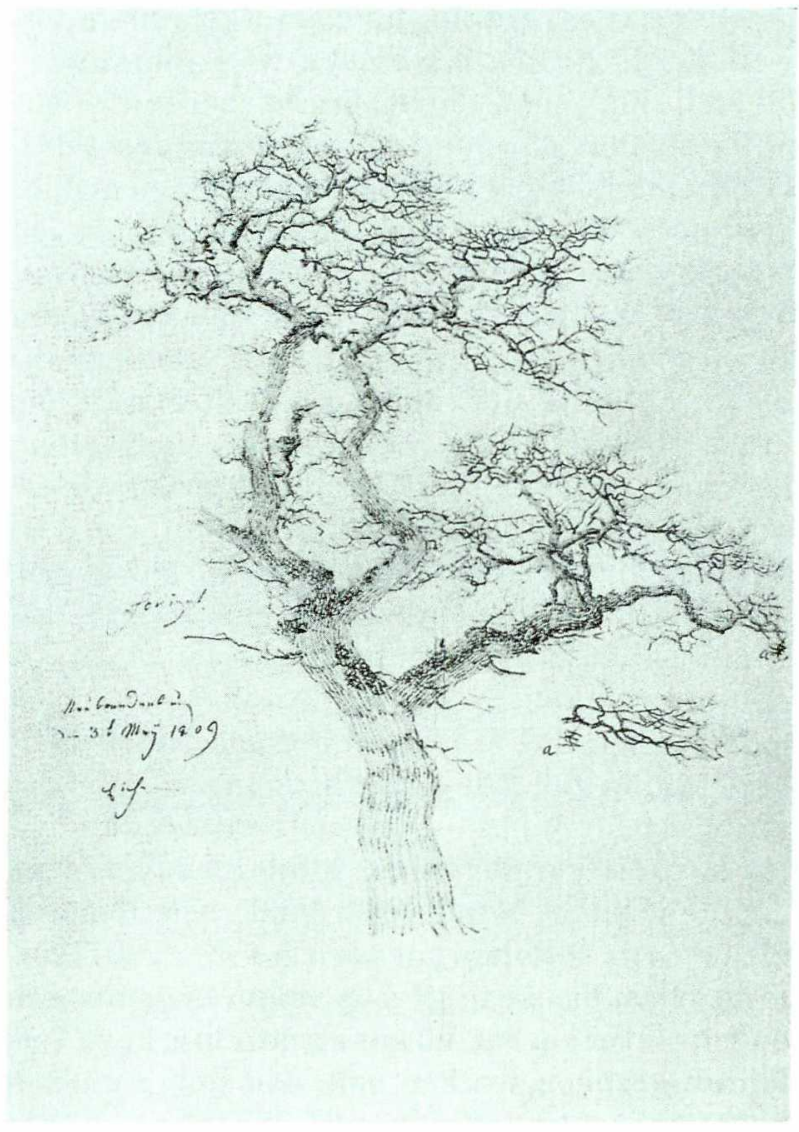

Abb. 2 Caspar David Friedrich, Entlaubte Eiche, Bleistift, 1809, $56,0 \times 25,9 \mathrm{~cm}$, Oslo, Nationalgalerie

staunen fest, daß die bei weitem häufigsten Studien Baumstudien sind. Und sucht man die Baumstudien nach Typen zu scheiden, so realisiert man, daß nur spärlich belaubte, wenn nicht kahle Bäume überwiegen. Nun kann man das, verkürzt gesagt, romantisch erklären wollen im Hinblick auf die Verwendung in Bildern. Der abgestorbene Baum als Vergänglichkeitsmetapher, der kahle als Zeichen finsterer Zeiten, im Winter erstarrt die Seele, die Hoffnung ist fern, ein leichtes Wiedergrünen markiert einen Hoffnungsschimmer. Das ist sicher nicht falsch, so oder ähnlich hat Friedrich die Baummetapher genutzt, auch vormärzlich etwa die nationale Eiche, die den Unbilden der Zeit trotzt. Doch erklärt dies weder die Art der Baumstudien, noch ihre große Zahl.

Betrachtet sei eine entlaubte Eiche, die Friedrich am 3. Mai 1809 bei Neubrandenburg gezeichnet hat (Abb.2). Sie folgt einerseits voller Demut dem Naturvorbild, jede noch so kleine Verästelung hat ihr Recht, auf dem Blatt zu erscheinen,

8 Otto Stoessl, Moritz von Schwind, Briefe, Leipzig 1924, S. 322 (an Franz von Schober, München, 5. Juni 1853). 
ja selbst als das Blatt sich für das ausgebreitete Astwerk der Eiche als zu schmal erwies, markiert er die Stelle mit einem kleinen Buchstaben und zeichnet gesondert darunter den Fortgang des Astes. Doch wie zeichnet Friedrich dies? Die Zeichenbögen haben zumeist, so auch hier, mittleres Format, etwa $36 \times 26 \mathrm{~cm}$, es wäre also durchaus möglich, mit dem Blei Flächen unterschiedlich starker Tönung zu wischen, in dem jeweiligen Ton Verlaufsmerkmale, Wölbung und Senkungen zu markieren. Friedrich tut dies nicht, vielmehr verwendet er ein paradoxes Verfahren der Charakterisierung: einerseits verzichtet er so gut wie nie darauf, die Wetterseite eines Baumes anzugeben - Verfärbungen, Moosansätze, Altersmerkmale -, andererseits läßt er die Gesamtform und diese Merkmale aus einem relativ abstrakten System von Parallelschraffuren hervorgehen, die, wie immer neu ansetzende Bahnen, Stamm und Äste bezeichnen, als würden damit Stufen im Wachstumsprozeß deutlich. Verdichten sich die Parallellinien, werden sie gekreuzt, dann entstehen besagte Merkmale. Die feineren kleinen Äste bestehen nur noch aus wenigen Parallelstrichen, bis sie in jeweils einem Bleistiftstrich enden. Bevor ein Schluß aus einem derartigen Verfahren gezogen werden soll, das sich natürlich auch, aber wirklich nur auch, bestimmten, vor allem Dresdener Zeichentraditionen verdankt (Zingg, Klengel), sei kurz ein anderer Friedrichscher Zeichentyp danebengestellt: der in Fernsicht gesehene Baumstreifen, quasi ein Stück Landschaft. Bezeichnenderweise gibt Friedrich manchmal mehrere derartige Streifen übereinander wieder, wie bei der Zeichnung, die er in Breesen am 9. Juni und am 12. Juni 1809 gezeichnet hat (Abb. 3). Der oberste Streifen mit den drei Eichen ist trotz des kleineren Maßstabes nach dem geschilderten Verfahren gezeichnet, hinzu kommt, daß das Blattwerk mit kleinen Strichelchen, aber ebenfalls parallel organisiert, festgehalten ist. Die Laubbaumstreifen darunter sind in größerer Entfernung genommen, nicht mehr der einzelne Baum in seinem Detail interessiert, sondern seine Figuration im Verhältnis zu anderen Baumfigurationen. Schaut man genau hin, so ist überraschenderweise der Umriß des einzelnen Baumes nicht etwa durch eine der Figuration folgende Linie markiert, sondern ergibt sich vielmehr aus dem Abbruch für sich gegenstandsunabhängiger Parallelschraffuren, bzw. dann wenn die Schraffuren, was nicht selten der Fall ist, von einer Figuration zu einer anderen durchlaufend gezogen sind, durch gleichmäßige Ab-

9 Eine entsprechende Reflexion zu einer künstlerischen Baumsprache zuerst bei Alexander Cozens, s. Busch, op. cit. (Anm. 4), S. 337-342. schwächung oder Verstärkung am Figurationsbeginn. Schließlich können die Parallelschraffuren so stark und dicht nebeneinandergesetzt erscheinen, daß sie das Dunkel zwischen versetzt gestaffelten Bäumen verkörpern, besonders deutlich beim untersten Landschaftsstreifen. Mit diesem graphischen System gelingt es Friedrich, den optischen Eindruck des Streifens überzeugend wiederzugeben. Doch nicht die Illusion ist entscheidend, sonst hätte er, was er bei den Studien nie tut - die bildmäßigen Zeichnungen sind sorgfältig davon zu scheiden -, laviert oder aquarelliert. Vielmehr geht es beim ersten wie beim zweiten Typus um das Festhalten von Strukturmerkmalen, für die, und das ist entscheidend, ein Flächenäquivalent gesucht wird, d. h. Friedrich denkt strukturell von der Bildfläche her.

Friedrichs ungezählte Baumstudien dienen der Vergewisserung einer künstlerischen Baumsprache, so daß bei der Integration der Studie ins Bild die im Bildgenerierungsprozeß sich ergebende Abweichung vom Naturvorbild dennoch naturrichtig wirkt. ${ }^{9}$ Dieser Vorgang unterscheidet sich vom klassischen Idealisierungsprozeß, der ein Generalisierungsproze $\beta$ ist. Hier bleibt die Individualität des Gegenstandes erhalten, sie ist jedoch letztlich eine subjektiv verfügte. Diese Dimension des Subjektiven schlägt sich im fertigen Bild nicht etwa als stehengelassene Faktur nieder, sondern im Gegenteil in einer gewissen Härte der Erscheinung. Bei aller Lasurarbeit im Atmosphärischen, für die Friedrich zu Recht berühmt war, so sind dennoch die einzelnen Gegenstände erkennbares Resultat analysierender Zeichnung. Diese Härte wiederum hat Schelling als notwendig begriffen. Die Kunst, so argumentiert er, erkennt im Individuum das ewige Urbild, »und wer das Wesen ergriffen, darf auch die Härte und Strenge nicht fürchten, denn sie ist die Bedingung des Lebens «. ${ }^{10}$ Die Härte bewahrt zwar das Individuelle, kehrt jedoch zugleich das als charakteristisch Erkannte hervor. Der Künstler, so Schelling, steigt ins Einzelne hinab, ja scheut selbst die Pein der Form nicht, um das Wesen der Werke der Natur zu entäußern. ${ }^{11}$ Bei aller philosophischen Abstraktion bezeichnet diese Bemerkung den praktischen Generierungsprozeß bei Friedrich sehr genau. Das hervorgekehrte Charakteristische, dessen Erkenntnis das zeichnerische Studium ermöglicht hatte, dient nun zugleich als eine Art Stilisierungsoder Verfremdungseffekt, an dessen Rändern, um es so auszudrücken, die romantische Verweisfunktion sich ansiedeln kann. Begreift man dies recht,

10 F. W. Schelling, Über das Verhältnis der bildenden Künste zu der Natur (1807), in: ders., Sämmtliche Werke, hrsg. von K. F. A. Schelling, Bd. 7, Stuttgart/Augsburg 1860, S. 304. 11 Ebd. 
so versteht man auch, daß der romantische Verweis nicht eigentlich im klassischen Sinn allegorisch ist oder auch nur sein kann - der Baum, der Fels, der Mond bedeuten nicht dieses oder jenes in stellvertretender Funktion, sondern sind individueller Baum, Fels, Mond, die zugleich in der Betonung ihrer strukturellen Dimension den Betrachter auffordern, die durch die Härte und Strenge erfolgende Isolierung und Fragmentierung der Gegenstände durch Zusammenhang- und Sinnstifung aufzuheben. Dieses Verfahren nennt Schelling im übrigen symbolisch. ${ }^{12}$

\section{ADOLPH MENZEL}

Viel ist zu Menzels besonderem Wirklichkeitszugriff, seinem permanenten zeichnerischen Aufnehmen des Gesehenen als einer Vergewisserung des Ist-Bestandes geschrieben worden, es soll hier nicht vermehrt werden, nur die Konsequenzen des alleinigen Vertrauens auf die authentische Erfahrung und ihre unmittelbare zeichnerische Fixierung seien anzudeuten versucht. Dieser Menzelsche Wirklichkeitszugriff ist subjektiv und objektiv zugleich zu nennen. ${ }^{13}$ Subjektiv, weil das Subjekt relativ frei darüber verfügt, welcher Gegenstand angeeignet wird, gleichgültig welchen Anspruch der Gegenstand in einer normativen Hierarchie der Gegenstände klassischer Auffassung nach auch verkörpern mag. Und objektiv, weil allein dem Erscheinungsphänomen Gerechtigkeit gegenüber geübt wird. Der Gegenstand mag erscheinen, in welchem Blickwinkel auch immer, so wie er erscheint, wird er festgehalten. Das führt zu einer Eroberung und zu einer Anerkennung der Bildwürdigkeit banaler Gegenstände, extremer Perspektiven, zum Festhalten von Posen, die nach klassischer Vorstellung unschicklich, bemüht, absurd, für den Dargestellten ungünstig, zufällig oder unerwünscht sein können. Es ist zumeist kein liebevoller Blick auf die Dinge, schnell, unbeteiligt, vom wahrgenommenen Phänomen unmittelbar gefordert, für eventuelle Verwendung gehortet, aber vielleicht auch schon für sich genug, abgebucht im Archiv der Selbstvergewisserung.

Menzels Authentizitätsbesessenheit macht nur Sinn, wenn man in ihr nicht allein ein Verfahren sieht, die Gegenstände genauestens kennenzulernen, sie in Bau und Funktion zu beherrschen, sondern wenn man begreift, daß es um die Bewältigung

12 Zu Schellings Symbolbegriff in bezug auf Friedrich s. vor allem: Joseph Leo Koerner, Caspar David Friedrich and the subject of landscape, London 1990, S. 144, besonders bezogen auf $\$ 39$ von F. W. Schellings Philosophie der Kunst (1802), in: ders., Sämmtliche

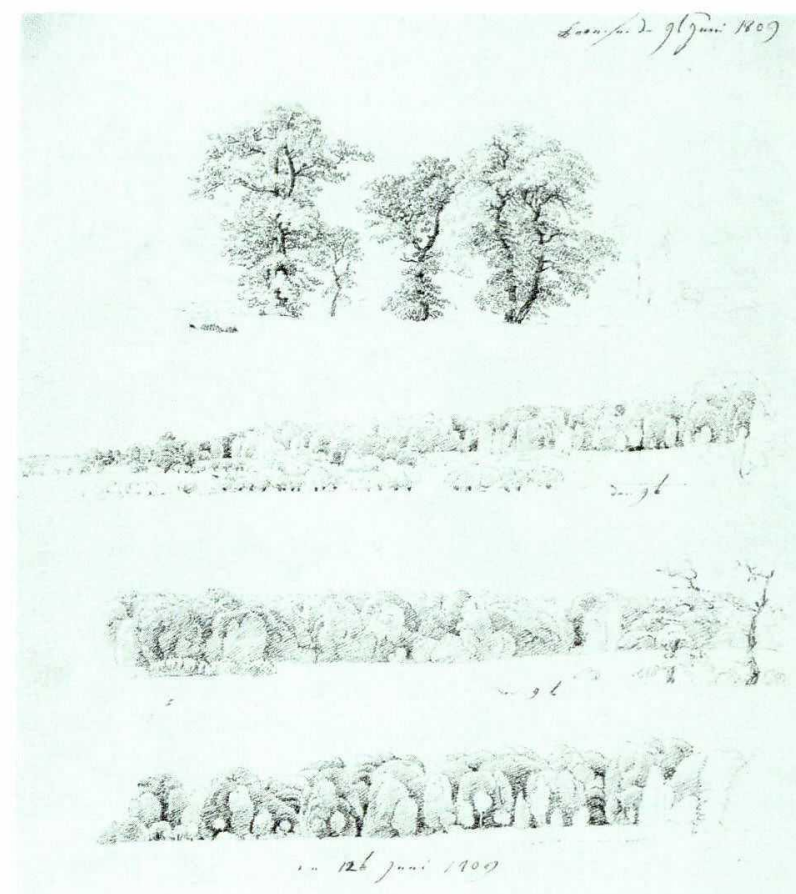

4bb. 3 Caspar David Friedrich, Baumstudien, Bleistift, 1809 , 36,2 × $25,7 \mathrm{~cm}$, Kunsthalle Bremen

der jeweiligen individuellen Sicht auf die Dinge geht. Es gibt eine Gruppe von Werken bei Menzel, die sich vom übrigen Euvre dadurch unterscheidet, daß zu Signatur und Datierung das Wort »Erinnerung « tritt. Dabei handelt es sich um eine seltsame Zwischengattung, zumeist um Pastelle, also eine Gattung zwischen Zeichnung und Gemälde, deren Zwischenposition auch im Gegenständlichen deutlich wird, denn es handelt sich zumeist nicht um wirklich Szenisches, schon gar nicht um komplexe Kompositionen, sondern um farbig weitergeführte Studien. Momentaufnahmen, die sehr weitgehend auf zeichnerische Studien zurückgehen und das zeichnerisch Erfaßte für wert erachten, weiterentwickelt zu werden. Erimnerung meint hier alleine, daß es sich nicht um eine unmittelbare Aufnahme vor dem Objekt handelt. Auf Menzels Skizzenbuchblättern finden sich an einzelnen Figuren oder Figurationen häufig kleine Kreuzchen, derartig gekennzeichnete Figurationen hält der Künstler für besonders gelungen und für weiter verwendbar. Nicht selten läßt sich ihre spätere Nutzung auch nachweisen. Mißlungenes streicht Menzel nachdrücklich durch. So dürften die Pastelle

Werke, hrsg. von K. F. A. Schelling, Bd. 5, Stultgart/Augsburg 1859 S. $406-413$

$13 \mathrm{Zu}$ dieser Spannung s. Werner Busch, Die notwendige Arabeske. Wirklichkeitsaneignung und Stilisierung in der deutschen Kunst des 19. Jahrhunderts, Berlin 1985, S. 282-286. 


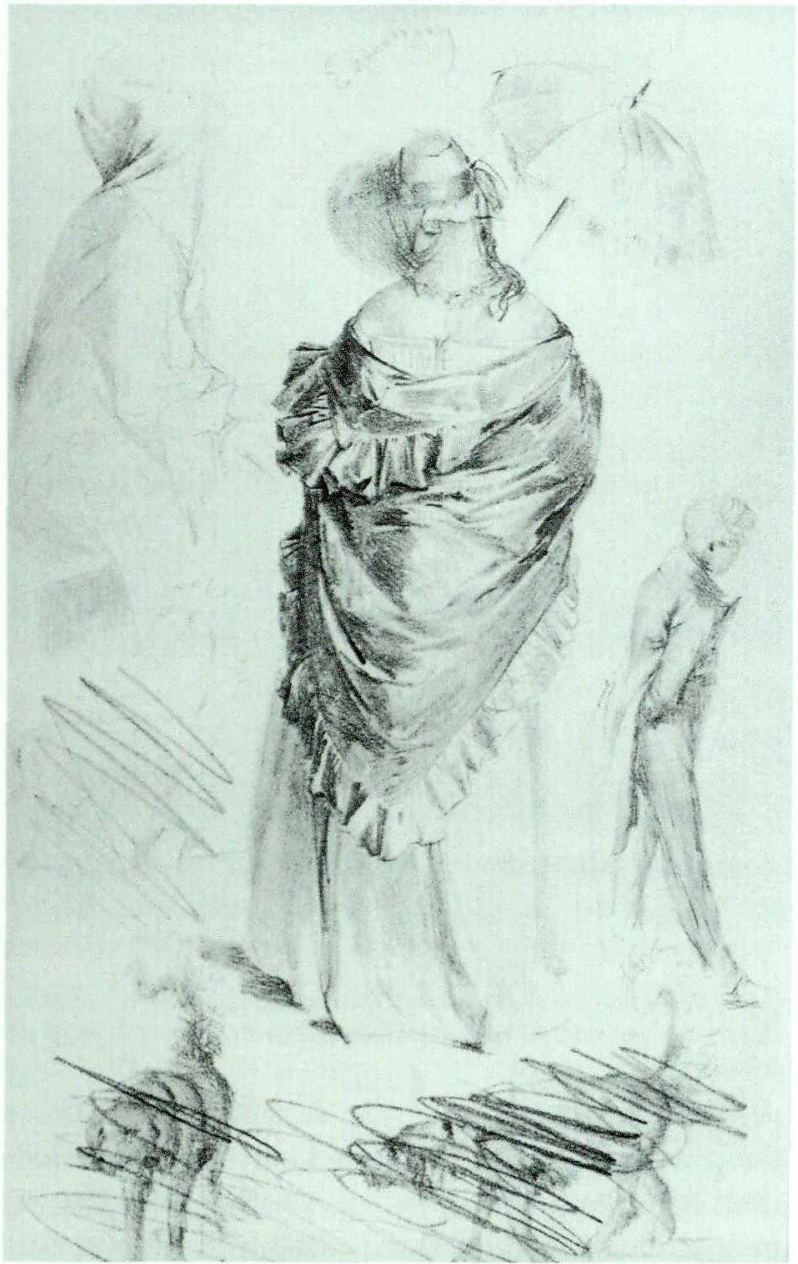

Abb. 4 Adolph Menzel, Studie aus dem Skizzenbuch von 1846 , Bleistift, 20,7 x 13,3 cm, Staatliche Museen zu Berlin, Kupferstichkabinett

erneute Vergegenwärtigungen gelungener zeichnerischer Notate darstellen. Im Skizzenbuch von 1846 findet sich nun allerdings auch eine Reihe von

14 Dies mag, allen anderen Vermutungen zum Trotz, der Grund für das Abbrechen der Arbeit an Menzels Historie »Ansprache Friedrichs des Großen an seine Generäle vor der Schlacht bei Leuthen sein, s. Kat. Ausst. Adolph Menzel, 1815-1905. Das Labyrinth der Wirklichkeit, hrsg. von Claude Keisch und Marie Lr-
Zeichnungen mit der Beischrift »Erinnerung (Abb. 4). In diesen Fällen dürfte es sich um sehr »kurzfristige« Erinnerungen handeln. Figürliche Motive, die beobachtend aufgefallen waren, aber nicht gleich festgehalten werden konnten, z. B. weil sie transitorisch waren, werden kurz darauf aus dem Gedächtnis nachgezeichnet. Kein Wunder offenbar, daß sich gerade in solchen Fällen gehäuft Durchstreichungen finden. Bei den »Erinnerungen« ist der Faden zum Gegenstand abgerissen und soll quasi nachträglich wieder befestigt werden. Für Menzel scheint es extrem wichtig, direkte Aufnahme und Erinnerung voneinander zu scheiden. Im Grunde genommen sind seine historischen Bilder Erinnerungen durch Rekonstruktion. Menzel erinnert sich an seine zeichnerischen Auseinandersetzungen mit den historischen Relikten in bestimmter Ansichtigkeit, baut aus diesen Wahrnehmungsnotaten und historischem Quellenstudium sein Bild. Der szenische Zusammenhang ergibt sich aufgrund der Wahrheit des Einzelnen im Idealfall von alleine, im negativen Falle scheitert das Bild. ${ }^{14}$

Erinnern wir noch einmal an Carstens und Friedrich. Carstens mochte auf die klassische Kunst rekurrieren, er wußte gleichzeitig, wie sein Freund Fernow überliefert, vom Vergangenheitscharakter der Kunst, von der erloschenen Gültigkeit der klassischen Themen in der Gegenwart. ${ }^{15}$ Friedrich setzte den Bruch mit der Vergangenheit absolut, vertraute allein auf die subjektive Erfahrung in der Gegenwart, der er auf ästhetischem Wege Dauer zu geben versuchte. Menzel schließlich rekonstruiert mit authentischen Partikeln, in der Hoffnung, den auch von ihm erfahrenen Bruch mit der Vergangenheit durch subjektive Aufnahme von alleine objektiv werden lassen zu können. Historistisch, d.h. aber auch modern, sind alle geschilderten zeichnerischen Verfahren gleichermaßen.

sula Riemann-Reyher, Nationalgalerie und Kupferstichkabinett, Staatl. Museen zu Berlin - PreuBischer Kulturbesitz, München 1997, Kat. Nr. 90.

15 Fernow, op. cit. (Anm. 5), S. 249-251. 